\title{
Using action research to develop human factors approaches to improve assembly quality during early design and ramp-up of an assembly line
}

\author{
J. Village*, F.A. Salustri, W.P. Neumann \\ Human Factors Engineering Lab, Mechanical and Industrial Engineering Department, Ryerson University, 350 Victoria St., Toronto, Ontario M5B 2K3, \\ Canada
}

\section{A R T I C L E I N F O}

\section{Article history:}

Received 8 October 2015

Received in revised form

1 April 2017

Accepted 24 May 2017

\section{Keywords:}

Human factors

Ergonomics

Production assembly design

Quality improvement

Design for assembly

House of quality

\begin{abstract}
A B S T R A C T
Engineers at a large electronics new product initiation site were interested in developing human factors (HF) approaches to help improve assembly quality during two stages of their production assembly design: early design of tasks, fixtures and tooling; and during early ramp-up of new assembly lines at outsourcing sites. Researchers worked in an action research approach with company engineers and ergonomists to integrate HF into both design stages. This paper presents the human factors approaches and discusses the challenges of using human factors to improve assembly quality. For the first stage of early design, a HF-design for assembly (HF-DFA) scorecard was developed with 22 items scored on a 0 (no risk or problem) to 2 (high risk or problems) scale. Items included physical risks, such as grip size and force, movement risks, such as re-grasping or re-orienting, visual risks, such as visual accuracy and inspection difficulty, and cognitive issues such as ability to detect a problem and risk of damage to part or component. High scores were associated with assembly tasks that were both reported as difficult by operators, and also had quality problems. The HF-DFA was adopted as a controlled engineering document and used to proactively score assembly tasks prior to final design of tasks, fixtures and tooling. In the second stage of early ramp, researchers combined the HF-DFA and other HF and performance-based metrics into a modified HF-house of quality (HF-HoQ) approach where the focus was on "worker" requirements rather than the traditional customer requirements. The HF-HoQ was evaluated using video of four identical tasks performed at different outsourcing locations that had a seven-fold difference in defect rates. The HF-HoQ successfully detected the site with the highest defect rate, but not the lowest. The authors recommend further testing and development of approaches that attempt to bring insight from $\mathrm{HF}$ to the issue of improving assembly quality.

Relevance to industry: Human factors is broader than injury prevention, and has been linked to assembly quality. Two HF approaches were developed to help improve quality in early design stages and during early ramp-up of assembly lines. Companies are encouraged to develop and evaluate HF approaches for improving assembly quality.
\end{abstract}

(c) 2017 Elsevier B.V. All rights reserved.

\section{Introduction and background}

Numerous studies have demonstrated an empirical link between human factors (HF, used here interchangeably with ergonomics) and production assembly quality and the issue has been discussed for many years (e.g. Zare et al., 2015; Drury, 1997; Eklund,

\footnotetext{
* Corresponding author. Present address: 2220 Badger Rd., North Vancouver, BC V7G 1T1, Canada.

E-mail address: jvillage@shaw.ca (J. Village).
}

1997). In a recent scoping review (Kolus et al., 2014), 73 studies were identified showing human factors (such as process instructions and training, product complexity, difficulty and load, and workstation conditions) were related to quality outcomes (such as frequency of failures or reworked and spoiled parts). The relationship between poor human factors and quality deficits was strongly supported throughout the processes of product design, production process design, and workstation design. In all, over 200 different HF variables were identified. In studies manipulating these variables effect sizes of over $85 \%$ impact on quality performance has been observed (Kolus et al., submitted). Kulos et al. 
(submitted) also noted in their review that fatigue as an underlying conceptual issue was mentioned in about half of all of these studies. Dode et al. (2016) used biomechanically modelled workload patterns to calculate a cumulative muscular fatigue dose which, in turn, accounted for about one quarter of the variance in all quality deficits in an electronics assembly operation. While improving quality is itself a valid reason to pursue arguments, the relationship between the HF quality risk factors and injury risk factors suggests this might pose a strategic lever for gaining support for HF from design teams that are held primarily responsible for system performance rather than worker safety (Neumann and Village, 2012; Neumann and Dul, 2010).

Most studies that evaluate the impact of human factors on quality defects identify injury risk factors in the tasks, and either (a) compare the extent of injury risk with the extent of quality defects, or (b) seek to reduce the risk and measure the effect on quality. For example, Falck et al. (2010) rated physical risk in automotive assembly jobs as high risk, medium risk, or low risk. The authors' reported increased quality errors in the high and medium risk jobs, compared with low risk jobs. Using a different approach, Gonzales et al. (2003) chose a metal production task with known ergonomic risk factors where quality metrics could be measured. The authors quantified the risk (using Rapid Upper Limb Assessment or RULA, McAtamney and Corlett, 1993) then reduced the ergonomic problems, re-scored the risks with RULA, and reported a $45 \%$ reduction in loss of materials (a quality measure) as a result (Gonzales et al., 2003). A study by Eklund (1995) utilized worker perceptions of ergonomic risk factors based on interviews about tasks with the most physical demands, most difficult parts to assemble, and the most psychological demands and found five times more quality deficits in tasks with ergonomic problems. These studies show that assembly tasks that are well designed from a human factors perspective will result in fewer quality problems. However, we could not find human factors tools or approaches that would help engineers detect quality problems or improve assembly quality directly.

\subsection{The need for human factors tools or metrics that can help improve quality during assembly design stages}

While many studies have successfully established a relationship between poor human factors and quality problems, the human factors (HF) indicators in all literature reviewed by the authors were based on injury-risk models, rather than other human factors issues directly related to specific aspects of quality (such as worker detection of quality problems, feedback about assembly fit, etc.). Additionally, the studies reviewed were all performed with injurybased tools on jobs, such as automotive assembly, that have high physical demands and therefore high magnitude risk factors. Further, most studies have been conducted in stable manufacturing operational environments, as opposed to during early design stages or early ramp-up of assemblies in manufacturing. Tools based on injury or physical demands cannot be used proactively in design stages to indicate quality problems because workers are not yet performing the tasks. Therefore, a HF tool with leading indicators is needed.

Instead of further proving a link between HF and quality, we were interested to improve understanding of how HF knowledge and approaches can be used directly to help engineers design with improved quality. Unlike automotive assembly tasks, we were working in an electronic assembly and manufacturing environment, where the physical risk factors of awkward and forceful posture are minimal. We were interested therefore in other $\mathrm{HF}$ indicators and metrics that could lead to improved quality (such as those addressing visual or tactile issues).
We were working in a new product initiation facility where assembly tasks, tooling, and fixtures were in early design stages for hand-held communication devices. Engineers we worked with were interested in ways of using $\mathrm{HF}$ information to help improve quality during two particular design stages. The first stage is early design of assembly tasks, tooling and fixtures for the small parts. Quality metrics are monitored very closely during this stage. Once design of tasks, tooling and fixtures is stable, those tasks, toolings and fixtures are sent to various outsourcing sites to begin mass production. The second stage of interest was therefore in early ramp-up and refining of the new assembly lines at the various outsourcing sites, where quality metrics can vary greatly.

\subsection{Case study collaboration, data collected, and objective of paper}

This paper uses a subset of data pertaining to quality and HF from a three-year longitudinal industry-university case study collaboration. The new product initiation site was in a large electronics manufacturer in Southern Ontario. The goal of the collaboration between ergonomists and engineers at the company, and researchers at Ryerson University, was to find ways to integrate human factors into the design of the assembly production system for both improved worker health and system performance. A full description of the collaboration and action research approach and methods are found in Village et al. (2014a,b). The data in this study has not been reported in the previous studies.

During the three years of collaboration, researchers worked with company ergonomists and engineers to use HF principles to help improve quality metrics in several initiatives. Interviews were conducted with quality engineers $(n=13)$ and floor supervisors $(n=2)$ to understand the nature of the quality data collected in the organization, how it was used, and who monitored it. Researchers and ergonomists attended meetings with engineers through a full production assembly design cycle $(n=22)$. They also participated in shop floor quality meetings as workers provided input to early problems assembling parts and using jigs, tooling and fixtures. The goal was to find ways to use HF tools and approaches to help improve aspects of product assembly quality. Table 1 summarizes the activities and number of company personnel participating in each activity over the three year period of the collaboration. Each activity was digitally recorded. Data was transcribed into NVivo software and coded qualitatively for general inductive analysis.

This case study is an in vivo illustration of how practical HF development occurs in organizations, and provides indications of what is missing in the HF research to help support such development work. The specific objectives of this paper are to:

1. Identify the challenges of using HF information to help improve a company's assembly quality in early design stages, and show how a customized tool, called the human factors design-forassembly (HF-DFA), might be created in vivo; and

2. Identify the challenges of using HF information to detect quality issues in early ramp-up of an assembly line, and report on a pragmatic approach called the HF house-of-quality (HF-HoQ) approach.

\section{Developing HF approaches to improve quality in two stages of production assembly design}

This section will present two in vivo attempts to develop HF approaches to improve assembly quality at two stages of the production assembly design. It will also present some of the limitations of using HF principles to improve quality of assembly in each stage. In the first stage - early design of the assembly tasks, tooling and fixtures - the goal was to help designers detect HF concerns related 
Table 1

Activities from 2010 to 2013 towards determining relationship between quality and HF.

\begin{tabular}{|c|c|c|c|}
\hline Year & $\begin{array}{l}\text { Number of } \\
\text { company } \\
\text { participants }\end{array}$ & $\begin{array}{l}\text { Number } \\
\text { Activities }\end{array}$ & Activity \\
\hline 1 (from Sept. 2010) & 10 & 10 & $\begin{array}{l}\text { Interviews with participants about quality and human factors, and how quality } \\
\text { data is organized in company }\end{array}$ \\
\hline 1 (from Sept. 2010) & 9 & 1 & Steering committee meeting where quality and HF was discussed \\
\hline 1 (from Sept. 2010) & 3 & 1 & Shop floor tour to discuss quality issues on line \\
\hline $2(2011)$ & 8 & 3 & Steering committee meetings where quality and HF was discussed \\
\hline $2(2011)$ & 3 & 3 & $\begin{array}{l}\text { Meetings with personnel in quality department to discuss data and how it } \\
\text { could be related to HF }\end{array}$ \\
\hline $2(2011)$ & 10 & 1 & Meeting with floor supervisors to discuss quality on new line \\
\hline $2(2012)$ & 20 & 2 & Two days ( $16 \mathrm{~h}$ ) data collection on ramp up of new line \\
\hline $2(2012)$ & 6 & 4 & Steering committee meetings where HF and quality discussed \\
\hline $2(2012)$ & 6 & 22 & $\begin{array}{l}\text { Meetings to discuss/present data from line, interpretation of quality data, } \\
\text { run DFA workshops }\end{array}$ \\
\hline $2(2012)$ & 4 & 4 & Meetings to do root cause analysis \\
\hline $3(2013)$ & 3 & 3 & Meetings to finalize DFA \\
\hline
\end{tabular}

Table 2

Task analysis comparison between four sites and Ergonomist comments.

\begin{tabular}{|c|c|c|c|c|}
\hline Steps & Site 1 & Site 2 & Site 3 & Site 4 \\
\hline \multirow[b]{8}{*}{ Comments } & 1. Pick up part in $\mathrm{RH}^{\mathrm{a}}$ & 1. Pick up part in $\mathrm{RH}$ & 1. Pick up part in $\mathrm{RH}$ & 1. Pick up part in $\mathrm{RH}$ \\
\hline & 2. Insert part in fixture & 2. Insert part in fixture & 2. Insert part in fixture & \\
\hline & 3. Pick up board in $\mathrm{LH}^{\mathrm{a}}$ & 3. Pick up board in LH (simultaneous) & 3. Pick up board in LH & 2. Pick up board in LH \\
\hline & 4. Pass to $\mathrm{RH}$ & & 4. Re-grip board using LH & 3. Hold and turn board in LH \\
\hline & 5. Lower board over part with $\mathrm{RH}$ & 4. Lower board over part with $\mathrm{LH}$ & 5. Lower board over part with $\mathrm{RH}$ & 4. Insert part while holding board in $\mathrm{LH}$ \\
\hline & 6. Insert connector with finger & 5. Insert connector with thumb & 6. Insert connector with tweezers & \\
\hline & 7. Make connection with tool & 6. Make connection with tool & 7. Make connection with tool & 5. Make connection with tool \\
\hline & $\begin{array}{l}\text { slower, taking care } \\
\text { finger then tool connection }\end{array}$ & $\begin{array}{l}\text { quick discrete motions } \\
\text { simultaneous } \mathrm{RH} \text { and } \mathrm{LH} \\
\text { thumb then tool connection }\end{array}$ & $\begin{array}{l}\text { fairly quick discrete motions } \\
\text { tweezer then tool connection }\end{array}$ & $\begin{array}{l}\text { appears slow but partly because no fixture } \\
\text { holding board and turning during insertion } \\
\text { perhaps better visually (closer) } \\
\text { no stability for making connection (holding) } \\
\text { only uses tool for connection (one step) } \\
\text { coordination appears more difficult }\end{array}$ \\
\hline
\end{tabular}

${ }^{\mathrm{a}} \mathrm{RH}=$ right hand; $\mathrm{LH}=$ left hand.

to quality prior to final design. To do this, researchers developed and piloted a new tool called the HF-design for assembly scorecard (HF-DFA). In the second stage - early ramp-up of assembly lines at different outsourcing sites - the goal was to help designers detect $\mathrm{HF}$ issues that may have been causing quality metrics to attain different values at different sites. Although all sites used identical assembly tasks, fixtures and tooling, the defect rates were found to vary more than seven-fold. In this case, researchers used various HF tools in a modified house-of-quality (HF-HoQ) approach to help detect the site with the highest quality defects. In this section of the paper, the challenges of each of the two attempts will be discussed, followed by an explanation of the development and testing of the tool or approach, and finally the lessons learned.

\subsection{Detecting $H F$ issues in early design stages and development of the HF design-for-assembly tool}

\subsubsection{The challenges}

Through interviews and observations, we found that $\mathrm{HF}$ issues at the new product initiation site tended to be detected towards the end of the development process during the early product builds when process, tooling and parts had been finalized, and just before product launch. The new product initiation site makes only small batches of the product, and therefore frequently missed problems that only become apparent under high volume production and time pressures. Unfortunately, this means that at times HF issues would not show up until the product is launched (ramped-up) at an outsourcing site and it is too late to affect major changes to production or component design, as described below:

Engineer: "With a recent product we had a difficult time latching the product together and fatigue time for operators was rather high. We identify this not so much in development - but when we begin to ramp - the demands to produce in the cycle time become more pronounced. We have to build to run it right - find issues of fatigue or operator difficulty - We go to bigger production numbers and the fixture starts causing hand pain we didn't see before."

There is widespread recognition amongst engineers of the importance of preventing $\mathrm{HF}$ issues prior to design of parts and assembly processes, but a $\mathrm{HF}$ tool or metric for assessing potential HF issues was missing. Production engineers attempt to identify issues that are "critical to quality" from past experience or specific product design features that may be problematic as indicated by this quote:

Quality Engineer: "From a HF perspective, we want early on [at design level] to make sure things are designed for manufacturability - this means we want to identify things that will be cumbersome to assemble, are going to be difficult to press manually, and ensure we are assembling in a certain action ( $x$ or $y$ direction) - we want to avoid assembling at an angle - we don't want awkward postures for the worker. Ideally we want to design it the best way first - but in some cases the look constrains us from a mechanical point of view and we're asked to assemble in all kinds of weird angles." 
The company engineers know from experience that "interference fits" (manual assembly of tight fits) can be an indicator of potential problems. They told us that if assemblies involved tight fits, operators started to feel pain in their fingers and hands, and defect rates would be higher. At other times too much force could cause pain, fatigue and injury, especially for manual fits that are performed repetitively. Too much applied force can also break parts, and too little can result in a poor connection. However, the goal of early detection of HF issues in product design, or even in early production design, is difficult without a HF tool or metric linked to quality. The light, repetitive assembly work observed in this organization had high visual demands, demands on attention to detail, fine motor skills, and sustained alertness. The researchers were unaware of a HF tool appropriate for scoring or rating tasks for these particular demands. One engineer asked what the human factor "metric" would be for an assembly step to be considered "good" or "safe." A pragmatic HF design-for-assembly tool was developed in response to these needs (described in next section).

\subsubsection{Development of the human factors design for assembly (HF- DFA) tool}

The HF-DFA tool is a practical example of how researchers developed a customized tool that could indicate potential human factors and quality issues early in design of the assembly process. This meaningful outcome of the collaboration was not planned in advance, but was identified as a need arising from several months of collaborative work between researchers, and company ergonomists and engineers through the complete assembly design process for a new product. Working through a complete process meant researchers initially participated in Failure Mode and Effects Analysis (FMEA) meetings for the new product to help detect and understand the quality concerns based on product drawings. We then participated in early part, fixture and tooling design. Once designed, researchers worked alongside engineers to observe operators perform assembly steps with new parts and fixtures. The researchers, based on this experience working with engineers and reviewing videotape of tasks, developed a 22 -item scorecard called the HF design-for-assembly (HF-DFA). Fig. 1 shows the full scorecard with definitions for each item. A simple scoring system was chosen since engineers requested that the tool be nimble and quick. Each item was scored as a " 0 " (no or minimal problem), " 1 " (some risk or problems), or "2" (high risk or problems) for a maximum score of 44 . Items included physical risks, such as grip size and force, movement risks, such as re-grasping or re-orienting, visual risks, such as visual accuracy and inspection difficulty, and cognitive issues such as ability to detect a problem and risk of damage to part or component.

To evaluate whether the items on the scorecard could predict assembly difficulty and quality problems, the researchers scored tasks on a new prototype assembly line and showed the resulting task scores to engineers and management. The tasks with high scores were tasks about which both engineers and workers had concerns. When the scores were shown to the Senior Director of Engineering, he stated "That's fantastic, I see a lot of value in this". He further suggested that this would be a great way to monitor improvements in new product initiation. Additionally, the Senior Director saw the tool as a way to give product designers feedback about their design. He stated: "we go back to the design team and say this is going to be hard to do, and they shrug their shoulders and say that's all we can do or it's the only way to do it." The Director believed the HF-DFA tool could help designers understand the significance of the HF problems. With support from the Senior Director of Engineering, we ran two focus groups with engineers to refine the scorecard and ensure the issues and wording were appropriate. The HF-DFA was then adopted into a controlled engineering document and established as one of four required targets (along with cost, defect rate, and yield) for which engineers were held accountable. Senior management agreed that no task should score a " 2 " on the HF-DFA, and that overall scores for each task should be continuously improved in the process optimization stage of the assembly build.

\subsubsection{Lessons learned about the HF-DFA}

The Senior Directors saw many advantages to this type of tool both as a way to quantify and monitor improvement in the new product initiation stages, and also as a way to communicate effectively with product designers, as mentioned in this quote:

Senior Director: "look at [x item to assemble] - we would have said [to product designers that] it would be challenging - but we can't give any relative ranking - we go back to design and say this is a problem - but we have nothing to show"

"this could quickly give build over build so we know "have I improved it?" - this addresses HF complexity - if I don't measure apples for apples [i.e. the same metric] I won't understand if the process is getting better"

The Directors also discussed the HF-DFA as an information source for their cost justification analyses, for example on whether to design a fixture or automate a task. One quality engineer stated that he could see the advantage of this tool and "it would have given me support during the discussion of whether to automate (a certain task that workers were having difficulty with)". The Senior Director described the impact of the HF-DFA on cost estimates as follows:

"[a task] may be a problem but it would cost very little to scrap - if we use the FMEA [for risk of quality problems], the HF-DFA [for ease of assembly], and also cost - we won't prioritize the wrong thing this tool will help rank and can be combined with cost - then business can prioritize - [it's a] standard output to normalize across teams"

The HF-DFA is a simple, purpose-built prototype tool customized to this assembly environment. While a traditional researcher may be concerned about such quick adoption when the tool had not formally been "validated" in studies, the engineers and management were unconcerned about this. During the focus groups, engineers observed videotape of a task and used the tool to score it. This gave some sense of the reliability of the tool between observers. However, this was not formally "tested" either. The Director was unconcerned about the lack of testing, stating that we can "design a tool, use the tool, and modify the tool" to continuously improve it. The value in the tool was its perceived ability to combine different aspects of ease of assembly into a score that could be used to prioritize tasks and drive continuous improvement. The Director suggested focusing first on tasks that are critical to quality, carry out an assessment at each workstation, and prioritize the ones with the highest scores. Researcher notes indicated that this was a very different approach for the Ergonomists; instead of focusing on the task with the highest risk factors for injury, they were now focusing on the engineer's most critical task for quality, and embedding some HF knowledge into task improvement. On subsequent assembly builds, the DFA Lead and the Ergonomist would score each task on a new assembly line together. Any tasks with a " 2 " were required to be improved such that the score was reduced. The Ergonomist worked with engineers towards finding a solution that would improve the score. This also opened the possibility to feed lessons learned back to product design engineers to 


\begin{tabular}{|c|c|c|c|c|}
\hline Task Consideration & 0 & 1 & 2 & Score \\
\hline 1. Grip time required & None & Short duration & $\begin{array}{l}\text { Sustained or repeated } \\
\text { actions }\end{array}$ & \\
\hline 2. Grip size and type & $\begin{array}{l}\text { Mid-range full } \\
\text { hand }\end{array}$ & $\begin{array}{l}\text { Small grip or slightly } \\
\text { outstretched }\end{array}$ & $\begin{array}{l}\text { Pinch grip or fully } \\
\text { outstretched hand }\end{array}$ & \\
\hline $\begin{array}{l}\text { 3. Re-grasping or re-orienting } \\
\text { hand or part }\end{array}$ & None & $\begin{array}{l}\text { Small amount } \\
\text { (1 re-grasp occasionally) }\end{array}$ & Considerable & \\
\hline $\begin{array}{l}\text { 4. Orientation variability: on pick- } \\
\text { up, placing or assembly }\end{array}$ & $\begin{array}{l}\text { Universal } \\
\text { orientation }\end{array}$ & Multiple orientations & One correct orientation & \\
\hline 5. Ease of placement first time & Consistent & Occasionally need to re-do & $\begin{array}{l}\text { Frequently need to re- } \\
\text { do }\end{array}$ & \\
\hline $\begin{array}{l}\text { 6. Force } \\
\text { (and/or resistance to movement) }\end{array}$ & None or low & Medium & High & \\
\hline $\begin{array}{l}\text { 7. Precision requirements } \\
\text { (consider alignment features and } \\
\text { angle) }\end{array}$ & None or low & Some precision required & $\begin{array}{l}\text { Considerable precision } \\
\text { required }\end{array}$ & \\
\hline 8. Hands required & One handed & $\begin{array}{l}\text { Second hand needed to hold } \\
\text { or guide }\end{array}$ & Two hands required & \\
\hline 9. Task coordination and motions & $\begin{array}{l}\text { Simple/Linear } \\
\text { (one motion) }\end{array}$ & $\begin{array}{l}\text { Two steps } \\
\text { (or motions) }\end{array}$ & $\begin{array}{l}\text { > Two steps } \\
\text { (or motions) }\end{array}$ & \\
\hline $\begin{array}{l}\text { 10.Impact to body due to } \\
\text { sharp/hard surface contact }\end{array}$ & None & Small amount & Considerable & \\
\hline 11. Wrist posture & Mid-range & $\begin{array}{l}\text { Slightly bent } \\
\text { (in any plane) }\end{array}$ & Considerably bent & \\
\hline 12. Shoulder posture (reach) & $\begin{array}{l}\text { Elbows close to } \\
\text { body }\end{array}$ & $\begin{array}{l}\text { Medium reach } \\
\left(<45^{\circ} \text { forward or side }\right)\end{array}$ & $\begin{array}{l}\text { Large reach } \\
\left(>45^{\circ}\right)\end{array}$ & \\
\hline 13. Shoulder loading & $\begin{array}{l}\text { None or arm } \\
\text { supported }\end{array}$ & $\begin{array}{l}\text { Holding weight of arm or } \\
\text { light part }\end{array}$ & $\begin{array}{l}\text { Holding weight of arm } \\
\text { and moderate to heavy } \\
\text { part/tool }\end{array}$ & \\
\hline 14. Task duration & Quick motion & Medium duration & Longer duration & \\
\hline 15. Visual requirements & Clear view & Partial obstruction & Blind assembly & \\
\hline 16. Visual accuracy & None or low & Some visual accuracy & High visual accuracy & \\
\hline $\begin{array}{l}\text { 17. Inspection difficulty } \\
\text { (during and after assembly) }\end{array}$ & None or low & Some inspection required & $\begin{array}{l}\text { High level of inspection } \\
\text { required }\end{array}$ & \\
\hline $\begin{array}{l}\text { 18. Visual Task Contrast } \\
\text { (from surroundings ) }\end{array}$ & Sharp/Distinct & Dull/Blending Tones & None/Matched Tones & \\
\hline $\begin{array}{l}\text { 19. Feedback of task success } \\
\text { (eg. snap or feel) }\end{array}$ & $\begin{array}{l}\text { Unnecessary or } \\
\text { obvious }\end{array}$ & Required but simple & $\begin{array}{l}\text { Required and easy to } \\
\text { miss }\end{array}$ & \\
\hline $\begin{array}{l}\text { 20. Ability to detect problem with } \\
\text { part (quality) }\end{array}$ & Obvious & Moderate & Difficult & \\
\hline 21. Fragility of part (quality) & Slight & Moderate & Significant & \\
\hline $\begin{array}{l}\text { 22. Risk of Damage to Part or } \\
\text { Component (quality) }\end{array}$ & Slight & Moderate & Significant & \\
\hline \multicolumn{4}{|c|}{ TOTAL HF-DFA SCORE (max 44) } & \\
\hline
\end{tabular}

Fig. 1. Human factors - design for assembly scorecard. 
HF DFA: Definitions and Comments

\begin{tabular}{|c|c|}
\hline 1.Grip time required & $\begin{array}{l}\text { - Longer duration (sustained }>5 \mathrm{sec} \text { ) and repeated gripping increases hand/arm } \\
\text { fatigue and injury risk }\end{array}$ \\
\hline 2. Grip size and type & $\begin{array}{l}\text { - Small pinch grips use smaller muscles that fatigue more quickly and have less } \\
\text { force } \\
\text { - Outstretched hands cannot generate force and precision }\end{array}$ \\
\hline $\begin{array}{l}\text { 3. Re-grasping or re- } \\
\text { orienting hand or part }\end{array}$ & $\begin{array}{l}\text { - Re-grasping increases muscle fatigue and time } \\
\text { - Multiple handling increase cosmetic quality risk }\end{array}$ \\
\hline $\begin{array}{l}\text { 4. Orientation variability } \\
\text { (on pick-up, placing or assembly) }\end{array}$ & $\begin{array}{l}\text { - Affects ease of task physically (posture), cognitively and visually as well as time } \\
\text { to perform task }\end{array}$ \\
\hline $\begin{array}{l}\text { 5. Ease of placement first } \\
\text { time }\end{array}$ & $\begin{array}{l}\text { - Affects muscle use/fatigue, time for task, potential errors and frustration } \\
\text { - Multiple attempts increases cosmetic and general damage }\end{array}$ \\
\hline $\begin{array}{l}\text { 6. Force (and/or resistance to } \\
\text { movement) }\end{array}$ & - Higher force (>1 kg for pinch grips) increases fatigue and injury risk \\
\hline $\begin{array}{l}\text { 7. Precision requirements } \\
\text { (consider alignment features and } \\
\text { angle) }\end{array}$ & $\begin{array}{l}\text { - Degree of accuracy affects posture, mental workload and time } \\
\text { - Considerable precision }+/-1 \mathrm{~mm} \text { accuracy in placement }\end{array}$ \\
\hline 8. Hands required & $\begin{array}{l}\text { - Two handed tasks increase shoulder load, coordination, precision requirements } \\
\text { and time, and potentially reduce quality }\end{array}$ \\
\hline $\begin{array}{l}\text { 9. Task coordination and } \\
\text { motions }\end{array}$ & $\begin{array}{l}\text { - Increased coordination affects muscle loading/fatigue, time and quality } \\
\text { - Two steps means inserting one side and needing to, for example, turn and } \\
\text { press or snap other side }\end{array}$ \\
\hline $\begin{array}{l}\text { 10.Impact to body due to } \\
\text { sharp or hard surfaces }\end{array}$ & $\begin{array}{l}\text { - Hard or sharp surfaces (on parts, materials, etc) can put pressure on } \\
\text { underlying nerves, arteries, and tendons } \\
\text { - Consider point of contact, size of surfaces, pressure and duration }\end{array}$ \\
\hline 11. Wrist posture & $\begin{array}{l}\text { - Mid-range (neutral) wrist posture associated with less muscle fatigue, higher } \\
\text { force capability and reduced injury risk }\end{array}$ \\
\hline $\begin{array}{l}\text { 12. Shoulder posture } \\
\text { (reach) }\end{array}$ & $\begin{array}{l}\text { - Increasing reach increases muscle load on shoulders and time to perform task, } \\
\text { and reduces precision }\end{array}$ \\
\hline 13. Shoulder loading & $\begin{array}{l}\text { - Work with unsupported arms increases shoulder load and fatigue and reduces } \\
\text { precision, especially with increased weight of a tool or part } \\
\text { - Reduced precision impacts quality and cycle time }\end{array}$ \\
\hline 14. Task duration & - Duration affects muscle loading, fatigue and time \\
\hline 15. Visual requirements & $\begin{array}{l}\text { - Poor visual requirements adversely affect posture of neck and arms and reduce } \\
\text { quality of assembly } \\
\text { - Obstructed assembly increases time }\end{array}$ \\
\hline 16. Visual accuracy & $\begin{array}{l}\text { - Visual accuracy }(<1 \mathrm{~mm}) \text { affects loading on muscles, mental workload and } \\
\text { visual demands/strain on worker }\end{array}$ \\
\hline $\begin{array}{l}\text { 17. Inspection difficulty } \\
\text { (during and after assembly) }\end{array}$ & $\begin{array}{l}\text { - Affects mental workload, time for task and potentially quality. } \\
\text { - May affect posture. }\end{array}$ \\
\hline 18. Visual Task Contrast & $\begin{array}{l}\text { - Lack of contrast increases visual requirements, and can lead to awkward } \\
\text { postures and operator error }\end{array}$ \\
\hline $\begin{array}{l}\text { 19. Task feedback } \\
\text { (eg. hear or feel snap) }\end{array}$ & $\begin{array}{l}\text { - Affects quality of assembly and mental workload } \\
\text { - Easy feedback improves cycle time }\end{array}$ \\
\hline 20. Ability to detect quality problem & $\begin{array}{l}\text { - Affects ease of assembly, and need for a work-around } \\
\text { - Affects repeatability and quality }\end{array}$ \\
\hline 21. Fragility of part & - Affects ease of handling, mental workload, cost/quality \\
\hline $\begin{array}{l}\text { 22. Risk of Damage to Part } \\
\text { or Component (quality) }\end{array}$ & - Affects cost/quality, mental workload \\
\hline
\end{tabular}

Fig. 1. (continued) 
inform the next new product design.

\subsection{Using HF information to detect quality issues in early ramp of an assembly line and development of the HF house-of-quality approach}

\subsubsection{The challenges}

The second design stage where engineers were interested in HF input to quality was the early ramp-up of the assembly lines at different outsourcing sites. As mentioned in section 2.1.1, many HFrelated problems were not detected until after the product had launched to outsourcing sites and was at full production volume. There were several roadblocks experienced by the researchers in trying to contribute HF expertise to reduce quality problems during early ramp-up. The first was determining what variables, HF or otherwise, were related to the quality problem. The second was determining which quality defect, among many, might have had some HF implications with which Ergonomists could assist. The third was tracing a quality defect back to a particular task and workstation to investigate the HF implications. These are discussed below.

2.2.1.1. Determining which variables were related to the quality problem. Although identical design of tasks, fixtures and tooling from the new product initiation site was provided to all outsourcing sites, there were product variances due to parts suppliers, worker training, and worker technique, among others. One Director discussed a situation where problems were not detected until months after ramp-up. In that case, part tolerances were out of specification with what the supplier provided in the development phase. Suppliers increase output from thousands to millions of units and their processes were not always capable of delivering consistent tolerances at those increased volumes. This increased assembly difficulty for operators and could cause subsequent pain and injury, alongside quality and yield variations. The following describes the situation:

Quality Engineer: "In the 5-10 days when ramping at outsourcing sites and thousands are handling products - parts coming from sub-suppliers may have been coming off one tool - but variability at ramp explodes 20-fold since [there are] many tools supplying parts - huge variability - operators are the ones that have to be adaptive to take into account variability of parts and fixtures - can be very cumbersome at times - some [parts] come together easier than others - for 4-5 weeks after - support folks are working hard to get rid of variability of parts - build counter measures into fixtures or tools or reduce variability at sub-suppliers - things eventually calm down and we can run serial production."

2.2.1.2. Determining which quality defect might have had HF implications. The company keeps an extensive database of more than 60 quality metrics for different segments of the assembly process. The researchers reviewed this database and worked with the quality engineers to understand the metrics, and identify which ones might relate to HF assembly issues. The researchers found that many quality metrics have no immediately apparent HF implications, but instead are a result of damage to parts or inconsistencies in manufacturing, such as "defective part", or "damaged part". Other quality metrics, such as "scratched part", or "damaged assembly", could have been the result either of part problems, or human interaction with the parts during assembly, but the data did not lend itself to determining which would be the case. Frequently, such as with the defect "scratched part", it was impossible to identify at which workstation or assembly task this defect may have occurred since several workers may have handled the part and caused the scratch. Only a few of the metrics could definitively be related to human interaction with the part, such as "missing component" or "misaligned assembly part". And, for some of these, the missing or misaligned part may not have been detected until several workstations later in the assembly.

2.2.1.3. Trying to trace a quality defect back to a specific task or workstation to make improvements. We found that most of the quality data in the organization was reported by product and line and could not usually be traced to individual workstations. Sometimes a product would pass four or five steps before a defect such as "connection not made" could be detected by a piece of test equipment. A further disconnect was that outsourcing sites organized workstation tasks in different ways. Some used more workers and shorter cycle times, while others use fewer workers and longer cycle times. Therefore, a task such as camera insertion might be performed at different workstations and in combination with different tasks, depending on the outsourcing site.

By contrast, most existing HF tools are designed to analyze risks for an individual worker at an individual workstation. Since we did not have quality data for each workstation, and workstation tasks varied at different sites, we had no way to compare a HF "score" or checklist with quality data at different outsourcing sites. Quality data was reported for a line, but researchers knew of no HF scores that could be applied to an entire line. Therefore it proved impossible to use HF data to help detect quality problems with the current quality measurement system in the organization.

\subsubsection{Development of a HF house-of-quality approach to detect quality problems}

2.2.2.1. Background. Our attempts to use HF information to help improve quality during early ramp-up at outsourcing sites led to a practical field study agreed to by the steering committee of the collaboration. In one case, the magnitude of quality discrepancies varied about seven-fold between four outsourcing sites for the same single connector task. The task was being performed differently at different locations leading engineers to suspect there may be detectable HF issues related to task performance. At some sites, the workers used a tool, while at other sites workers performed the task manually. There were also different manual processes used. The quality problem was either that the connection was not made, or that the connection would break, resulting in scrap. The connection was measured directly and interactively at the assembly step and was either a binary "pass" or "fail."

The organization provided video of the task being performed by workers using different methods at the four locations. The goal was to see whether HF information could be used to predict which location and methods had the highest and lowest defect rates. This was a "blind" study, in that researchers were blind to the defect rates in each site. The goal was to evaluate different HF tools to potentially reveal the best HF metric(s) that could be related to quality for future detection of $\mathrm{HF}$ issues. Note that researchers did not have any organizational information pertaining to the sites, such as working hours, work-rest breaks, job rotation, etc. With the recent success of the HF-DFA tool, researchers were interested in whether the tool could predict the site with the most quality problems. We were also interested in whether other ergonomics tools could be helpful to predict quality problems. We therefore used the HF-DFA and other selected ergonomic tools in an adapted house-of-quality approach, since the house-of-quality is a recognized industrial engineering approach that combines methods to compare between competitors. 
2.2.2.2. Method of developing the HF house of quality (HF-HoQ). A house of quality (HoQ) diagram (see Fig. 2) is a quality function deployment tool that helps determine whether a product meets customer needs by connecting needs to engineering requirements and product properties, and allows comparison of the ability of different products to meet customer needs. We modified the house-of-quality method such that we could compare task performance across the four company locations from the perspective

\section{EXHIBIT X}

\section{House of quality}

\section{CUSTOMER} ATTRIBUTES

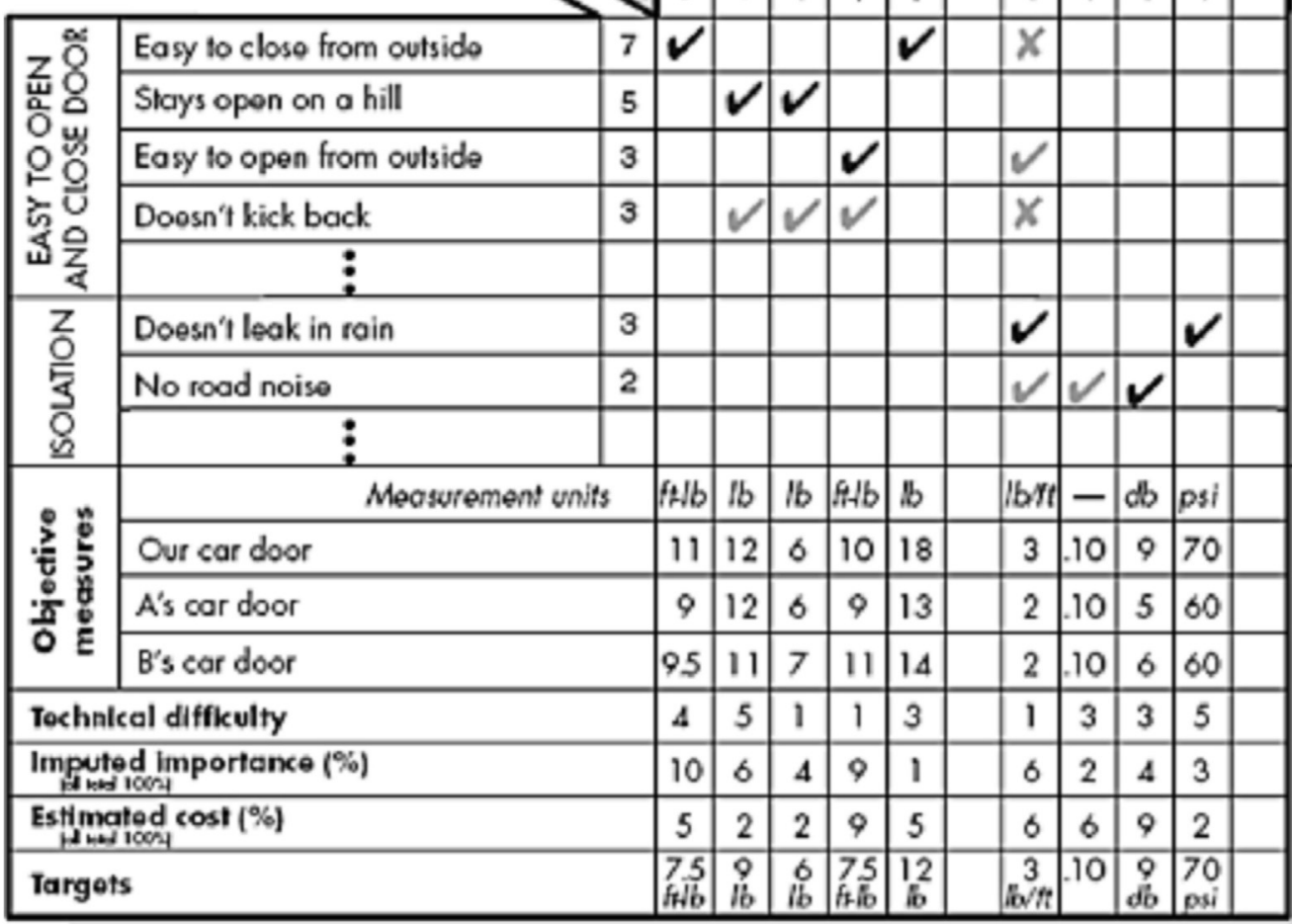

\section{Relationships}

$\checkmark$ Strong positive

$\checkmark$ Medium positive

$X$ Medium negative

X Strong nogative

\section{Customer perceptions}
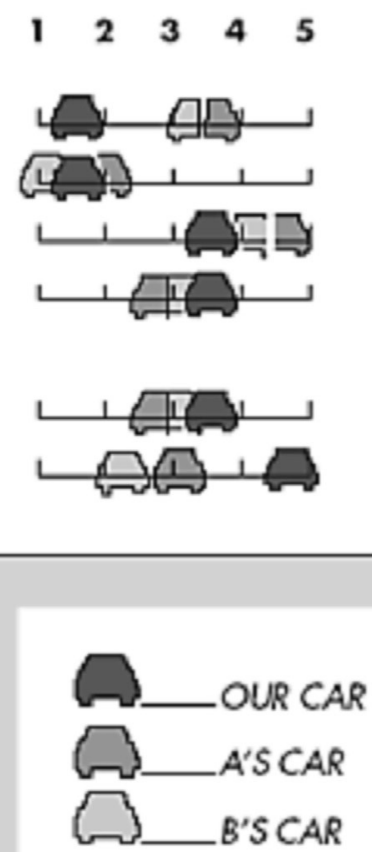
of "operator requirements," rather than "customer requirements." We hypothesized that if HF issues are improved for operators, then quality will also be improved.

Fig. 2 shows the original HoQ (Hauser and Clausing, 1987). The centre portion of the HoQ shows how customer (or user) attributes are related to engineering properties; notice that hierarchical organizations of these properties is possible. The "roof" of the HoQ captures coupling between the engineering properties. The right side allows one to document the performance of competitor's products with respect to customer attributes (placing primacy on the "voice of the customer" over the engineering preferences). The "basement" of the HoQ is reserved for project management measures. Overall, the information captured in a HoQ is intended to help make robust and evidenced product design decisions; it is not in and of itself a decision-making method.

To develop our HF-modified HoQ we had to translate the standard HoQ to accommodate our needs in this case. The translation is fairly straightforward. Customer attributes become "operator requirements" and the engineering requirements become the "production requirements" for the workers' station(s). Since our goal is to facilitate comparison between different sites, we use the right-side "competitors" submatrix of the regular HoQ to document differences between the different sites; i.e., we think of the different sites as "competing" with each other for the best combined quality and HF achievements. Finally, we use the "basement" to capture key project management characteristics of relevance to the case.

The first step in fleshing out our HF-HoQ analysis was to identify the HF tools and metrics supported by scientific literature, that would be most important to "operator requirements" (as opposed to the traditional focus on "customer" requirements). To choose the metrics, we reviewed the videos of workers performing the task and reviewed possible factors that could affect quality, such as number of assembly steps, timing of assembly steps, cycle time, complexity of assembly steps, available vision, parts presentation, and workstation support. We then searched for measures to quantify each of these (results discussed below).

To further understand the quality risk factors, we performed a root cause analysis to identify failure modes and possible causes. A root cause analysis is a tool to identify what, how and why something happened in order to prevent its recurrence. Fig. 3 shows the root cause analysis performed to trace the potential defects in this task, like "connection not made," with possible HF reasons such as space, force, lack of auditory feedback, etc. This exercise performed with HF Specialists together with engineers helped identify HF issues that might not otherwise have been identified by engineers alone. The risk factors discussed during this exercise helped inform the choice of methods for comparing the HF issues at the four sites.

A task analysis was then conducted to compare the number and types of assembly steps, and their time to completion. Table 2 shows the basic task analysis highlighting the different methods used by workers at the four sites. The first three sites all used a fixture to hold the part, while the fourth site did not. Therefore the part was stabilized on the work surface in the first three sites and held by the worker above the work surface at the fourth site. Different methods and combinations of methods were used at the different sites to make the connection including a finger, a customized tool, a thumb, and tweezers. The connection time varied significantly as noted in the comments section of the task analysis in Table 2.

2.2.2.3. Results using the HF-House of quality (HF-HoQ). As shown in Fig. 3, for the HF-HoQ the researchers chose the five most appropriate ergonomic criteria for the hand-arm task that could be measured given the available information, as the: HF-DFA score, the strain index (Moore and Garg, 1995), the threshold limit value (TLV) of the American Conference of Governmental Industrial Hygienists (Latko et al., 1997), cycle time as a percent of motion time methods (MTM) predicted task time, and number of assembly steps. Data for these criteria was used to evaluate the four sites, and added to the HF-HoQ (see Fig. 4).

Recall that our goals are to rank order the sites and use the information in the HF-HoQ to explain how that ranking was achieved. In this way, we expect to discover what characteristics, processes, and behaviours might be transferred from "best" sites to improve performance of all the sites.

Analysis of results with the new HF-DFA tool designed to score ease of assembly showed site 4 had the highest scores, regardless of which method of ranking was done (single highest hand score, averaged scores for both left and right hands, or combined right and left hand scores). The lowest risk was scored as site 3 when using the averaged or combined scores. The strain index rates speed of motion, force, posture, numbers of motions and the duration of motion to create an overall multiplied score. Regardless of whether the scores are combined or highest score is used, site 2 and 3 were the highest risk (and therefore presumably, the highest defect rates). The TLV uses a repetition scale for hand activity in combination with a predicted hand force to yield a composite score. In comparing sites using the TLV, site 2 followed by site 3 had the highest risk of injury.

In using cycle time as a percent of MTM time, we made the assumption that more time taken to perform the task would mean better quality. We used the motion time analysis system to predict the task time based on the detailed task analysis of the steps. We compared the cycle time as a percent of MTM time to indicate whether workers were performing above or below the predicted speed of the assembly task. Both site 1 and 4 were performing above the predicted MTM time and sites 2 and 3 were performing below the predicted. Regarding the number of assembly steps, we assumed that more steps for an operator would be preferable since that would provide the operator more variety of motions. Increased variety of motions should reduce the physical strain and boredom associated with a smaller number of highly repetitive motions. Site 2 had the lowest number of steps and therefore higher risk, and site 1 had the highest number of steps and therefore lowest risk.

The researchers also used the HF-HoQ to compare production requirements for the assembly task (which might not match "human" requirements). These were assumed to be short cycle times, short connection times, and a short time per assembly step. The researchers subjectively rated the importance of the five criteria on a 1-10 scale, consistent with the house-of-quality methodology. The HF-DFA was rated highest (9) as we believed it came closest to measuring the $\mathrm{HF}$ and quality issues pertinent to the task. We scored the strain index and TLV as an 8 given their validation in the scientific literature for hand-arm injury. We scored cycle time as a percent of MTM-predicted time an importance rating for the operator of 6. Finally, we scored the number of assembly steps as a 5. The relationship between these five criteria and production criteria are assumed small if indicated with a triangle (score of 1 ), medium if indicated by a clear circle (score of 3 ) and strong if indicated by a bold circle (score of 9 ). The score comparison is the product of the importance ratings and the relationship ratings. The HF-DFA has no direct criteria that are time-based, although it implies that a task that is easier for the operator to assemble would also be faster to perform.

Fig. 3 shows the comparative ranking across sites for the five criteria, as well as the sum and overall HF ranking (with 1 being highest HF risk ranking and 4 being lowest). Fig. 3 also shows the ranking of defect rates for the four sites, the "basement" of the diagram (with 1 being the highest defect ranking and 4 being 


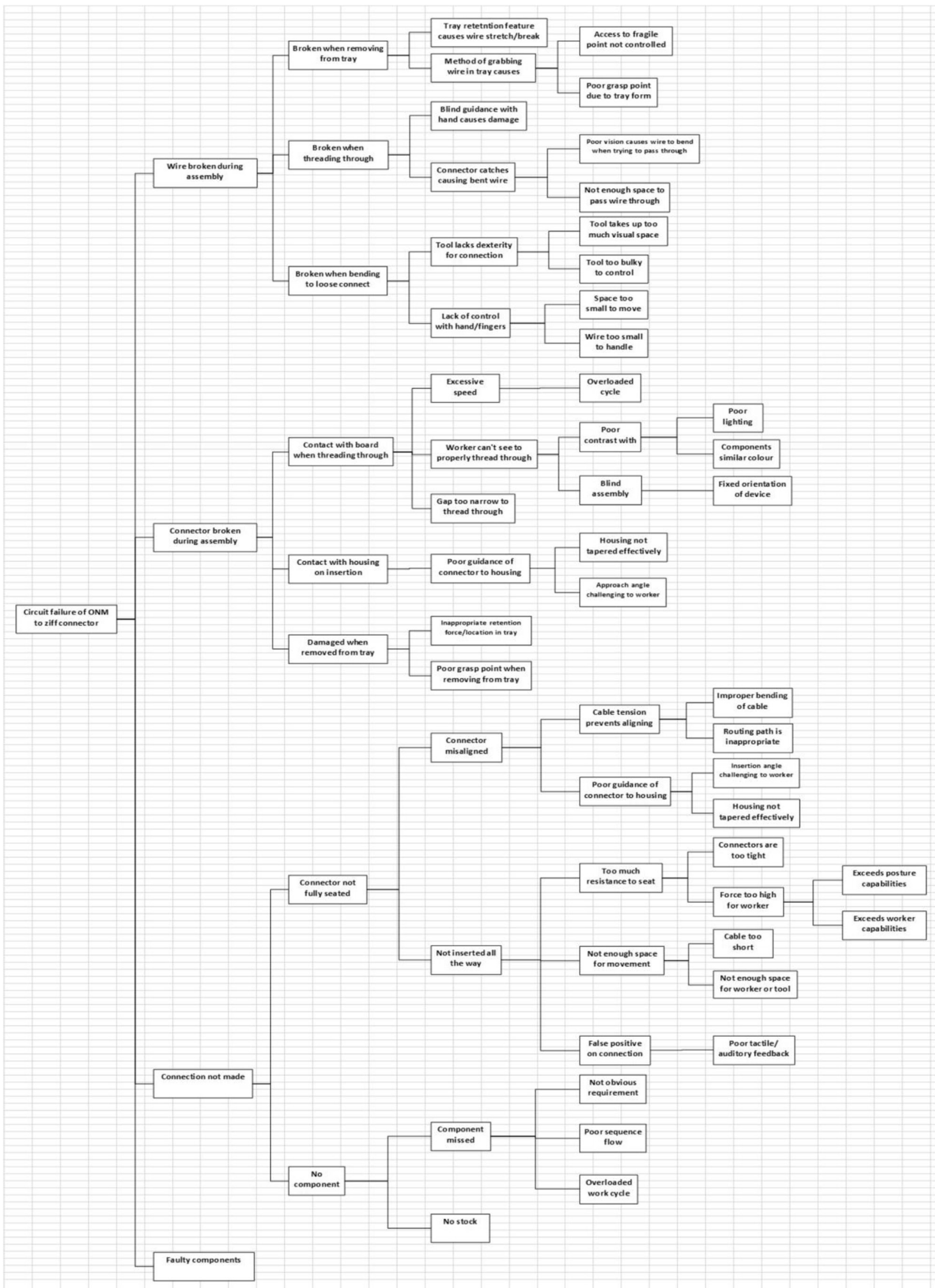

Fig. 3. Root cause analysis of possible HF-related quality problems. 


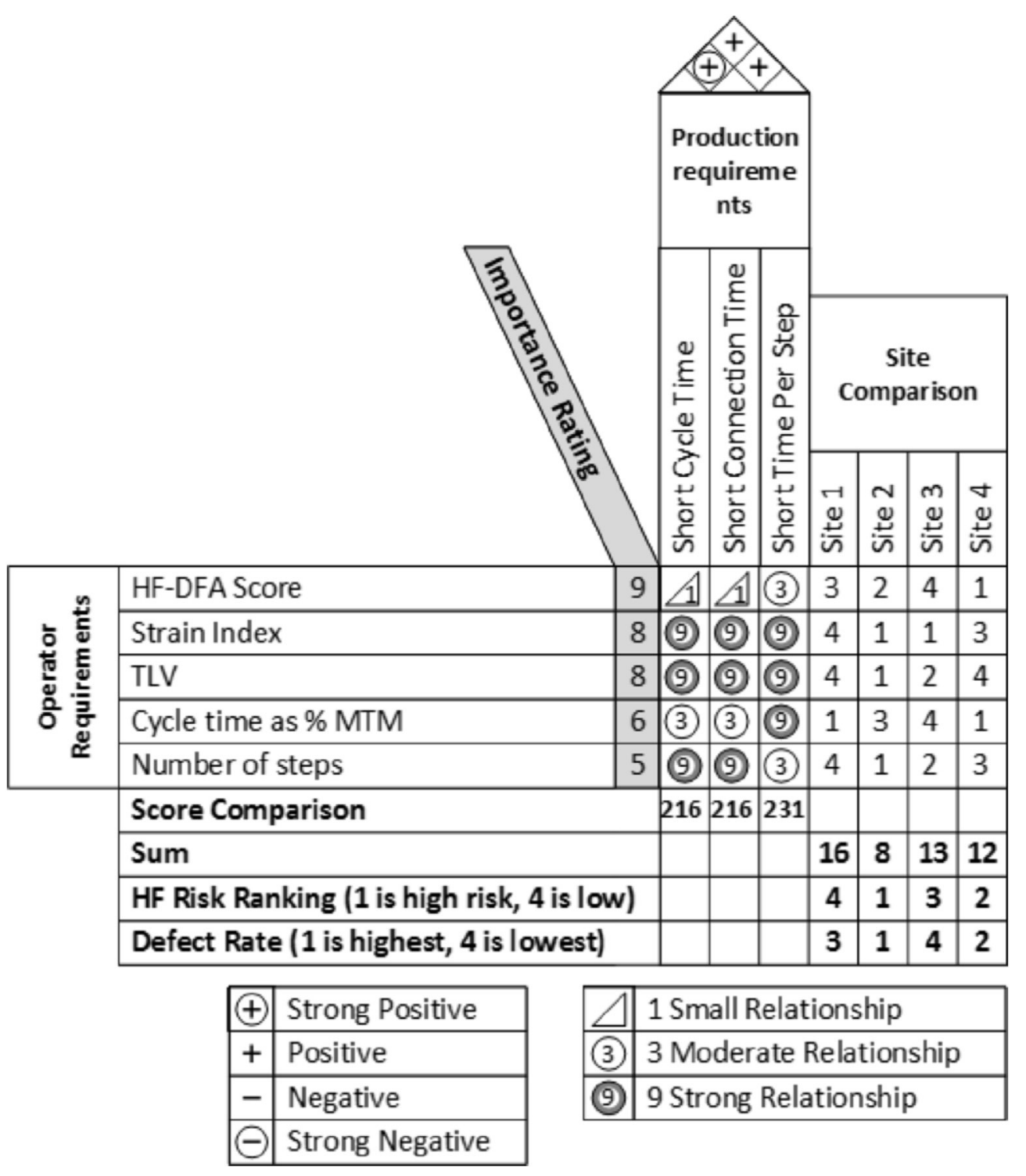

Fig. 4. Modified House of Quality comparing Operator and Production Requirements.

lowest). The HF-HoQ ranking successfully matched the highest defect rating (site 2). However, the lowest defect rate (site 3 ) was not successfully matched with our overall ranking, which we suggested was third. The HF-DFA seemed reasonably able to distinguish the differences between defect rates with the highest defect rate site scoring a two, and the lowest defect rate site scoring a four. The strain index and TLV scored both high and low defect sites similarly. Cycle time as a percent of MTM-predicted time seemed sensitive to the low defect rate site (rating of four), but not to the highest defect rate site (rating of 3). Conversely, the number of steps was sensitive to the high defect rate site (rating in 1 ), but not the lowest defect rate site (rating of 3). The strength of a house of quality analysis is that several criteria, rather than a single criteria, are used and weighted according to perceived importance. In this example, we were able to detect the site with the highest defect rate, but not the site with the lowest.

\subsubsection{Lessons learned about the HF-HoQ}

In the absence of a single HF assessment tool validated for detecting quality problems, we modified the house-of-quality approach by using the HF assessment tools and other criteria that we believed would be most applicable to HF and quality issues in the specific tasks in this light assembly environment. The composite HF-HoQ approach was then used in a blind comparison of tasks to see if it could detect the task with the highest and lowest defect rates. Although we were able to correctly detect the site with the highest defect rate, the approach did not correctly detect the site with the lowest defect rate. There are numerous assumptions made when developing a HoQ approach which would change the numbers and affect the results. Despite this, the approach has face validity, and certainly more use and testing is suggested. Further laboratory experimentation with different metrics and tasks could yield a more refined HF-HoQ tool applicable to this environment. The process of conducting the detailed task analysis and root cause analysis leading up to the criteria developed in the HF-HoQ provided insight and discussions about the task that were useful in thinking about HF issues related to quality. We suggest further investigation with this HF-HoQ type of approach as a potential tool that combines expertise from both the HF and engineering domains. 


\section{Discussion: how can HF help improve quality?}

Despite the company having numerous very specific quality metrics available for each product assembly line during new product initiation and at outsourcing sites, we were unable to determine which defects may be related to human factors issues at the workstation level and therefore where to focus our HF efforts to help improve quality metrics. Eklund (1995) also found difficulty using quality data directly in their study so they used inspector "judges" to make estimates about defect rates to compare with jobs considered most demanding. Eklund (1995) discussed challenges of using quality statistics, including that they were seen as estimates, are based on subjective judgements, sometimes have vague criteria or changing definitions, and can have different measurement points. As in our study, Eklund (1995) questioned where the quality data should be measured - at the assembly workstation, at subsequent inspector workstations, in final adjustment, after leaving the line, at the dealer, or at the customer. With current HF tools designed for workstation-level assessment, and quality data collected at the level of the assembly line in this company, researchers could not predict which HF issues were related to which defects. If the company were to collect quality data at each workstation, it may lead to better detection of quality problems, and better linkages with HF data.

Researchers in this study could not find a validated tool or metric for scoring HF issues that might be related to quality defects. Most HF tools score a worker/workstation interaction for risk factors related to musculoskeletal injury. However, while some defects may be related to worker strain and physical fatigue, others may be related to cognitive or visual demands, or to the complexity of the assembly steps. This is consistent with the dominant view in Canada that ergonomics is primarily concerned with occupational health and safety, and not with productivity or quality issues (Theberge and Neumann, 2013). It could be that a measure such as duty cycle (relationship between work and rest) may be better correlated with quality problems, but this has yet to be studied. Erdinc and Vayvay (2008a, 2008b) developed a method called "QUITE" (quality improvement through ergonomics) that suggests using the most appropriate ergonomic method for before-and-after assessment of a task after HF improvements. This approach was reported to be successful in stable manufacturing environments. However, in the rapid new product initiation process, there are too many variables changing all the time and too little time for this type of before-and-after assessment approach. Also, we needed tools that could proactively measure the risk of quality problems from $\mathrm{HF}$ issues prior to design of parts and fixtures and without the need for an operator to perform the tasks. In some assembly environments, an error proofing technique called "poka-yoke" is used by engineers to minimize human error in assembly. It would be interesting to investigate the extent to which this technique also makes the assembly easier and safer for workers. This investigation has highlighted the need for more research to yield the HF determinants of quality to be used in early design stages. As Falck and Rosenqvist (2012) has shown, $60-70 \%$ of HF issues arise from decisions made at the product design stage, and the remaining $30-40 \%$ are due to poor process design decisions (design of workstations, work organization, etc.).

Due to a lack of HF tools or metrics that could detect quality problems in early design or early ramp stages of assembly design, our objectives were to develop and describe two context-specific tools: 1. the HF design for assembly (HF-DFA) tool that combined issues related to human factors, as well as potential quality problems; and 2. the HF house-of-quality (HF-HoQ) tool to detect HF issues early in ramp as a consequence of HF and production demands. Some of the features in our HF-DFA tool were similar to findings of Falck and Rosenqvist (2012), especially geometryrelated errors of assembly such as hidden assembly, poor assembleability, and combined force and precision demands. The HF-DFA tool was developed "in vivo" with participation of engineers (manufacturing, industrial, quality, mechanical) in the organization who have extensive experience with designing assembly parts, components, tasks, tooling, fixtures and jigs. Village et al. (2014a,b) report other HF-adapted tools also designed in a participatory way with engineers in this company. Despite the lack of "formal" or "rigorous" testing, the tool had sufficient face validity that it became a controlled engineering target for continuous improvement inside the company. It also proved a useful tool, used in conjunction with other tools such as cost and FMEAs, to provide justification for changes to parts or tooling during new product initiation. As Buckle (2011) points out, "The perfect is the enemy of the good". In other words, does one wait for the "perfect" research evidence before embarking on practice, and how much evidence is "good enough" for action? Senior managers in this organization saw the value of the HF-DFA tool and were prepared to use it in a continuous improvement fashion to improve assembly design. They were also prepared to continuously improve the tool as new information, different problems, or lessons learned warranted. The development of this tool using a participatory approach by internal experts is most certainly generalizable to other industries and assembly environments.

Despite extensive experience among the researchers viewing the videos, there was little scientific basis with which to compare the different operator methods (finger connection, tweezers, tool) in order to predict difficulty, or strain and therefore associated quality problems. The researchers adopted a quality improvement approach, using a modified HF House of Quality method, to make informed choices about the most applicable HF knowledge and tools (such as the ACGIH TLV and strain index), in combination with engineering design criteria (number of assembly steps, and cycle time as a percent MTM-predicted time), and our new HF DFA to compare the four tasks. We made assumptions based on the speedaccuracy trade-off. That is, if operators are working faster, and operators have less variety, errors will increase. When sites were ranked using each of the five criteria and combined into an overall ranking of HF risk, it detected the site with the highest defect rates, but did not predict the site with the lowest rate. This unique approach may have merit, and we recommend the need for further study using this or other similar approaches. There are research and practice opportunities for engineers and ergonomists to work together to investigate which aspects of a task (vision, force, geometry of assembly, speed, etc) lead to quality problems. There are also opportunities to investigate ways to help operators learn to improve their detection of errors. For example, the design of aids to help workers know if an assembly step has been completed properly, such a screw that is fully seated or has been missed.

We recommend that ergonomists work with engineers to help improve quality in companies, but they need to change their focus from one of detecting tasks with a high risk of injury, to a focus on tasks that are critical to quality. This shift in perspective is the main driver in the "Design for Human Factors Grounded Theory" that explains how ergonomists acclimated and aligned human factors to help achieve design and business goals in this case study (Village, et al., 2015). Ergonomists can help manufacturing and design teams assess critical to quality tasks from the operator perspective (eg. difficulty to perform, visual requirements, tactile feedback, etc) and can bring a different perspective to finding solutions to minimize quality defects. Ergonomic assessments can then become a tool for how to help the operator maintain ease of assembly, consistency, and high detection of quality problems. 


\section{Conclusion}

More HF tools and approaches are needed to help detect and reduce assembly quality problems related to worker performance. The objectives of this study were to adapt and customize two approaches. The HF-DFA is a 22-item scorecard for early detection of assembly problems during design of tasks, fixtures and tooling. It's ease of use, face validity, and reported capability at detecting difficult to assemble tasks resulted in it being adopted as a controlled engineering document and required target in the assembly design process. The development of this tool has potential generalizability to others looking to customize a DFA tool. The HFHoQ is a unique adapted approach using various HF and performance tools to compare ease of an assembly task from an operators' perspective across different outsourcing sites. When used in a blind comparison of four sites, it successfully rated the site with the highest defect rate, but not the one with the lowest. More testing and evaluation of these and other HF-adapted tools is recommended such that HF can be applied beyond injury reduction, to improving assembly quality and system performance. While limited to a new product initiation site for small hand-held electronic products, the approaches discussed may stimulate others to incorporate $\mathrm{HF}$ into the assembly design process for the purposes of helping to improve quality metrics.

\section{Acknowledgements}

The authors would like to acknowledge the funding support from the Natural Sciences and Engineering Research Council and the Workplace Safety and Insurance Board in Ontario.

\section{References}

Buckle, P., 2011. 'The perfect is the enemy of the good' - ergonomics research and practice. Institute of ergonomics and human factors annual lecture 2010. Ergonomics 54 (1), 1-11.

Dode, P., Greig, M., Zolfaghari, S., Neumann, W.P., 2016. Integrating human factors into discrete event simulation: a proactive approach to simultaneously design for system performance and employees' well being. Int. J. Prod. Res. 54 (10), 3105-3117. http://dx.doi.org/10.1080/00207543.2016.1166287.

Drury, C.G., 1997. Ergonomics and the quality movement. Ergonomics 40 (3),
249-264.

Eklund, J., 1995. Relationships between ergonomics and quality in assembly work. Appl. Ergon. 26 (1), 15-20.

Eklund, J., 1997. Ergonomics, quality and continuous improvement - conceptual and empirical relationships in an industrial context. Ergonomics 40 (10), 982-1001.

Erdinc, O., Vayvay, O., 2008a. Quality improvement through ergonomics methodology: conceptual framework and an application. Int. J. Prod. Qual. Manag. 3 (3), $311-324$.

Erdinc, O., Vayvay, O., 2008b. Ergonomics interventions improve quality in manufacturing: a case study. Int. J. Ind. Syst. Eng. 3 (6), 727-745.

Falck, A.-C., Ortengren, R., Hogberg, D., 2010. The impact of poor assembly ergonomics on product quality: a cost-benefit analysis in car manufacturing. Hum. Factors Ergon. Manuf. Serv. Ind. 20 (1), 24-41.

Falck, A.-C., Rosenqvist, M., 2012. What are the obstacles and needs of proactive ergonomics measures at early product development stages? - an interview study in five Swedish companies. Int. J. Ind. Ergon. 42, 406-415.

Gonzales, B.A., Adenso-Diaz, B., Torre, P.G., 2003. Ergonomic performance and quality relationship: an empirical evidence case. Int. J. Ind. Ergon. 31, 33-40.

Hauser, J., Clausing, D., 1987. The house of quality. BBR 66 (3).

Kolus, A., Wells, R., Neumann, W.P., 2014. A framework for describing the relationship between human factors and quality. Proceedings of the Association of Canadian Ergonomists (ACE) 45th Annual Conference, Montreal

Kolus, A., Wells, R. P., Neumann, W. P. Production quality and human factors: A systematic review and theoretical framework, Int. J. Oper. Prod. Manag., (submitted).

Latko, W.A., et al., 1997. Development and evaluation of an observational method for assessing repetition in hand tasks. Am. Ind. Hyg. Assoc. J. 58 (4), 278-85.

McAtamney, L., Corlett, E.N., 1993. RULA: a survey method for the investigation of work-related upper limb disorders. Appl. Ergon. 24, 91-99.

Moore, J.S., Garg, A., 1995. The Strain Index: a proposed method to analyze jobs for risk of distal upper extremity disorders. J. Am. Ind. Hyg. Assoc. 56, 457-458.

Neumann, W.P., Dul, J., 2010. Human factors: spanning the gap between om \& hrm. Int. J. Operations Prod. Manag. 30 (9), 923-950. http://dx.doi.org/10.1108/ 01443571011075056.

Neumann, W.P., Village, J., 2012. Ergonomics action research ii: a framework for integrating hf into work system design. Ergonomics 55 (10), 1140-1156.

Theberge, N., Neumann, W.P., 2013. The relative role of safety and productivity in canadian ergonomists' professional practices. Relat. Industrielles/Industrial Relat. 68 (3), 387-408.

Village, J., Greig, M., Zolfaghari, S., Salustri, F., Neumann, W.P., 2014a. Adapting engineering design tools to include human factors. IIE Trans. Occup. Ergon. Hum. Factors 2, 1-14.

Village, J., Greig, M., Salustri, F., Zolfaghari, S., Neumann, W.P., 2014b. An ergonomics action research demonstration: integrating human factors into assembly design processes. Ergonomics 57 (10), 1574-1589.

Village, J., Searcy, C., Salustri, F., Patrick Neumann, W., 2015. Design for human factors (DfHF): a grounded theory for integrating human factors into production design processes. Ergonomics 58 (9), 1529-1546.

Zare, M., Croq, M., Hossein-Arabi, F., Brunet, R., Roquelaure, Y., 2015. Does ergonomics improve product quality and reduce Costs? A review article. Hum Factors Ergon. Manuf. Serv. Ind. http://dx.doi.org/10.1002/hfm.20623 n/a-n/a. 